\title{
Influence of Coriolis force on bulk flows during horizontal Bridgman growth on a centrifuge: a numerical study
}

\author{
W.J. Ma ${ }^{\mathrm{a}, 1}$, B.C. $\mathrm{Khoo}^{\mathrm{b}}$, D. $\mathrm{Xu}^{\mathrm{b}, *}$ \\ a Institute of Mechanics, Chinese Academy of Sciences, Beijing 100080, People's Republic of China \\ ${ }^{\mathrm{b}}$ Institute of High Performance Computing, 89 Science Park Drive, \# 01-05/08, The Rutherford, Singapore Science Park 1, Singapore 118261
}

Received 24 January 1998; accepted 28 May 1998

\begin{abstract}
Three-dimensional and time-dependent numerical simulations are performed for melt convection in horizontal Bridgman crystal growth under high gravity conditions by means of a centrifuge. The numerical results show that Coriolis force can cause a stabilizing effect on the fluctuations of the melt flow under a specific rotation direction and rotation rates of the centrifuge as reported in previous experiments (Ma et al., Materials Processing in High Gravity, Plenum Press, New York, 1994, p. 61). The present simulation provides details of the flow features associated with the effect of the Coriolis force. There are also some differences between the present three-dimensional and former twodimensional numerical solutions particularly in the prediction of the critical conditions and flow patterns. (C) 1998 Elsevier Science B.V. All rights reserved.
\end{abstract}

PACS: 81.10.F8; 02.60. +y; 47.30. +s; 47.15.Rg; 44.25. +f

Keywords: CFD simulation; Bridgman growth; High gravity; Crystal growth

\section{Introduction}

Crystal growth from the melt is an important way to produce a variety of materials. In the melt growth techniques, one of the main problems is the formation of a spatial type of micro-inhomogeneities (striations) in the crystals. Unsteady

\footnotetext{
*Corresponding author. Fax: + 165775 0092; e-mail: cmxud@leonis.nus.edu.sg.

${ }^{1}$ Currently working at the National University of Singapore as a visiting scientist.
}

buoyancy-driven convection occurring in the melt has been considered to be the most important reason leading to temperature fluctuation in the melt and resulting in the formation of the doping striations [2]. Recent studies have shown the possibility of growing homogeneous crystal by using a centrifuge. But the mechanism and underlying physics have not been fully understood.

Rodot et al. [3,4] found that the silver distribution in PbTe becomes uniform when the material is solidified in a centrifuge at a particular rotation rate. Müller et al. $[5,6]$ has shown that the use of 
centrifuge can help to avoid the problems associated with the formation of striations and other types of nonuniformity. They performed Te-doped InSb crystal growth experiments utilizing either the inverted vertical Bridgman technique or the horizontal zone melting method.

A GaAs crystal was first grown successfully using the horizontal Bridgman technique on a large centrifuge $[7,8]$. Dopant striations were found in the resulting crystals; these striations, however, become much less dominant and indistinct as the centrifugal acceleration is increased and the rotation sense of the centrifuge is coincident with the direction of the bulk convection roll in the liquid phase.

Some numerical simulations of bulk flows have also been attempted to confirm the above-mentioned experimental findings [9-11], with detailed simulations for the configuration of the vertical Bridgman growth. On the other hand, only preliminary two-dimensional simulations were performed for the configuration of horizontal Bridgman growth. These numerical studies have shown the possible importance of Coriolis force. It can be suggested that the presence of the Coriolis force, rather than "high gravity", is the critical stabilizing influence on the unsteady buoyancy convection, and eventually leads to the improvement of the quality of grown crystals.

In this paper, it is our intention to present the results of three-dimensional numerical modeling of bulk flows in a horizontal Bridgman growth configuration for various Grashof numbers in time-dependent flow regimes. The effect of Coriolis force on the bulk flow is specially taken into consideration, and its effect on the flow melt is analyzed in detail.

\section{Solution strategy of the numerical simulation}

\subsection{Description of the physical model and numerical modeling}

Horizontal Bridgman growth on a centrifuge is schematically depicted in Fig. 1 (From Ref. [11]). A horizontal cylindrical furnace is positioned at the end of the arm via a bearing with its axis perpendicular to the centrifuge arm and earth gravity. An

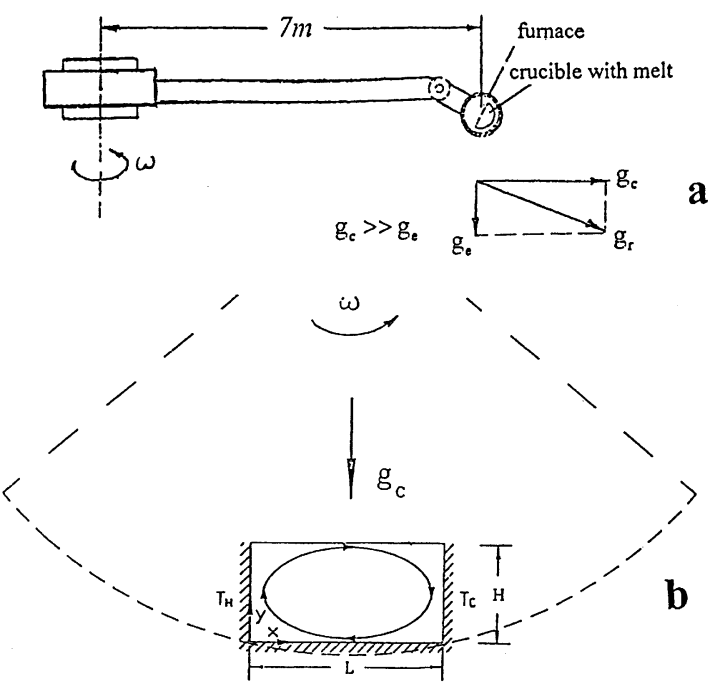

Fig. 1. (a) Sketch of crystal growth by the horizontal Bridgman technique on a large centrifugal; (b) schematic illustration of the top view, the crucible is simplified as a rectangular parallelepiped with enlarged size to show the inside flow (from Ref. [11]).

open-boat containing a molten metal is placed in the furnace; in Fig. 1a, the cross-section of the crucible is drawn, and the "vertical" section can be seen in Fig. 1b. For simplicity and in view that the main physics of the flow will not be unduly affected, the crucible in the present simulation is modeled as a rectangular parallelepiped with free surface at the top. The melt is heated by the furnace resulting in a horizontal linear temperature profile; in essence, a thermal buoyant convection is generated with the flow ascending at the hot end and descending at the cold end.

In general, the purpose of employing a centrifuge for crystal growth is to provide an acceleration field significantly larger than earth gravity $\boldsymbol{g}_{\mathrm{e}}$ with the centrifugal acceleration greater than $g_{\mathrm{e}}$ by at least a few times. In experiments, it is not uncommon to note that the ratio of centrifugal acceleration to earth gravity is of about an order; for example in Refs. $[1,7,8]$, this ratio ranges from 6 to 14 and more. To numerically simulate such a flow in line with the large centrifuge experiments, the magnitude of centrifugal acceleration is kept to be in the range larger than $6 \boldsymbol{g}_{\mathrm{e}}$. Even at the low limit where the centrifugal acceleration is $6 \boldsymbol{g}_{\mathrm{e}}$, the crucible will 

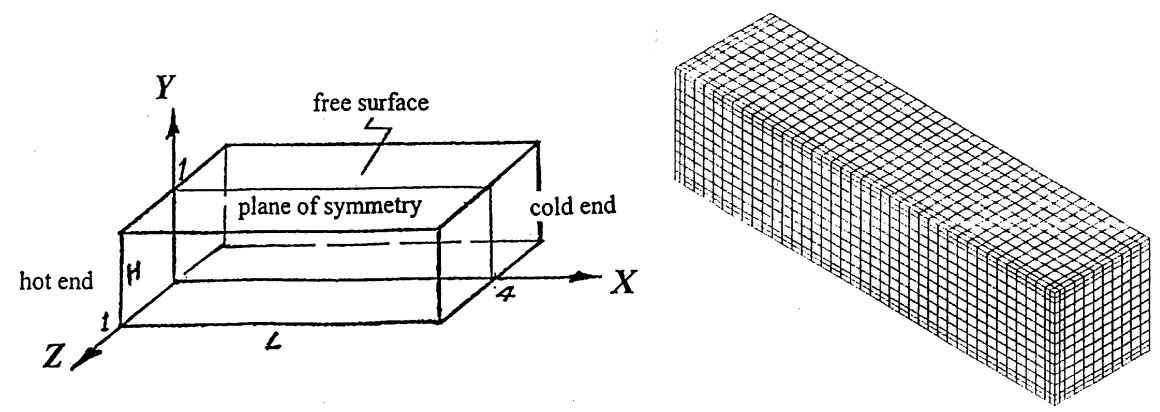

Fig. 2. Geometry of the problem and the mesh employed.

lie on a position near the plane of the centrifuge with an angle less than $10^{\circ}$, and the angle decreases with larger centrifugal acceleration. Therefore, in the present work, the buoyancy can be regarded as dominated by the centrifugal acceleration; the influence of gravity can be neglected with the primary physics of the flow remains largely unaffected. As a consequence, the centrifugal acceleration is taken to be perpendicular to the melt surface.

With the neglect of the earth gravity, there is a plane of symmetry extending from the "hot" to the "cold" sides of the crucible, as shown in Fig. 2. Fig. 2 also provides the geometry of the problem with a typical mesh of $(41 \times 13 \times 11)$ in the $x-y-z$ coordinate system representing the melt flow in the half-rectangular shaped crucible which measures $4 H \times H \times H$ used in the computation. Here $H=0.01 \mathrm{~m}$ and the rotating arm of the centrifuge is taken as $7.0 \mathrm{~m}$ reflecting the typical dimensions found in experiments $[1,7,8]$.

In this study, it is reasonable to assume that the flow is driven primarily by buoyancy caused by the combined action of the horizontal temperature gradient and the centrifugal acceleration $\boldsymbol{g}_{\mathrm{c}}$. The Marangoni convection is ignored. The centrifugal and Coriolis acceleration effects are separately treated in terms of nondimensional parameters $\mathrm{Gr}$ (Grashof number) and Ta (Taylor number), respectively.

\subsection{Governing equations and boundary conditions}

The continuity equation, Navier-Stokes equation with Bossinesq approximation and the energy equation describing the melt flow in the crucible are given as follows [2]:

$$
\begin{aligned}
\nabla \boldsymbol{u}=0, & \\
\boldsymbol{u}_{\mathrm{t}}+(\boldsymbol{u} \nabla) \boldsymbol{u}= & -\frac{1}{\rho} \nabla \boldsymbol{p}+v \nabla^{2} \boldsymbol{u}+\beta \boldsymbol{g}_{\mathrm{r}}\left(\boldsymbol{T}-\boldsymbol{T}_{\mathrm{C}}\right) \\
& -2 \omega \times \boldsymbol{u}, \\
T_{\mathrm{t}}+(\boldsymbol{u} \nabla) T= & \kappa \nabla^{2} T .
\end{aligned}
$$

Here $\boldsymbol{u}$ is the velocity vector, $p$ the pressure, $v$ the kinetic viscosity, $\beta$ the coefficient of volumetric expansion, $T$ the temperature, $\omega$ the rotation rate and $\kappa$ the thermal conductivity. $g_{\mathrm{r}}$ is the resultant gravity, i.e. $\boldsymbol{g}_{\mathrm{r}}=\boldsymbol{g}_{\mathrm{e}}+\boldsymbol{g}_{\mathrm{c}}$ (see Fig. 1a). $\boldsymbol{g}_{\mathrm{e}}$ is earth gravity and $\boldsymbol{g}_{\mathrm{c}}$ is centrifugal acceleration. The last two terms on the RHS of Eq. (2) represent the buoyancy force and Coriolis force, respectively.

Next, the following nondimensional variables (with prime) are introduced:

$\boldsymbol{r}^{\prime} \frac{1}{\boldsymbol{H}} \boldsymbol{r}, \quad t^{\prime}=\frac{\sqrt{\mathrm{Gr}} v}{\boldsymbol{H}^{2}} t, \quad \boldsymbol{u}^{\prime}=\frac{\boldsymbol{H}}{\sqrt{\mathrm{Gr}} v} \boldsymbol{u}$,
$p^{\prime}=\frac{\boldsymbol{H}^{2}}{\sqrt{\mathrm{Gr}} \rho v^{2}} p, \quad T^{\prime}=\frac{T-T_{\mathrm{C}}}{T_{\mathrm{H}}-T_{\mathrm{C}}}$.

The governing equations can then be re-written in nondimensional form as:

$$
\begin{aligned}
& \nabla \boldsymbol{u}=0, \\
& \boldsymbol{u}_{\mathrm{t}}+(\boldsymbol{u} \nabla) \boldsymbol{u}=-\nabla p+\frac{1}{\sqrt{\mathrm{Gr}}} \nabla^{2} \boldsymbol{u}+\boldsymbol{e}_{\mathrm{g}} T \\
& -\frac{\sqrt{|\mathrm{Ta}|}}{\sqrt{\mathrm{Gr}}} \boldsymbol{e}_{\omega} \times \boldsymbol{u} \\
& T_{\mathrm{t}}+(\boldsymbol{u} \nabla) T=\frac{1}{\operatorname{Pr} \sqrt{\mathrm{Gr}}} \nabla^{2} T,
\end{aligned}
$$


where $\boldsymbol{e}_{\mathrm{g}}$ and $\boldsymbol{e}_{\omega}$ refer to the unit vectors in directions of the resultant gravity $\boldsymbol{g}_{\mathrm{r}}$ and the rotation velocity vector of the centrifuge $\omega$, respectively. Here the prime (') corresponding to the respective dimensionless variables has been omitted for simplicity. As discussed in Section 2.1, in line with the experiments in a large centrifuge $[1,7,8], \boldsymbol{g}_{\mathrm{c}}$ is taken to be in a range larger than $\boldsymbol{g}_{\mathrm{e}}$ by six times or more. Therefore, the latter is neglected such that $\boldsymbol{e}_{\omega}$ will then be parallel to the $z$-axis (see Figs. 1 and 2).

The nondimensional parameters in the governing equations are the Prandtl number

$\operatorname{Pr}=v / \kappa$,

which is set to be 0.02 in the present study; the Grashof number

$\mathrm{Gr}=\left|\boldsymbol{g}_{\mathrm{r}}\right| \beta\left(T_{\mathrm{H}}-T_{\mathrm{C}}\right) H^{3} / v^{2}$

(a)
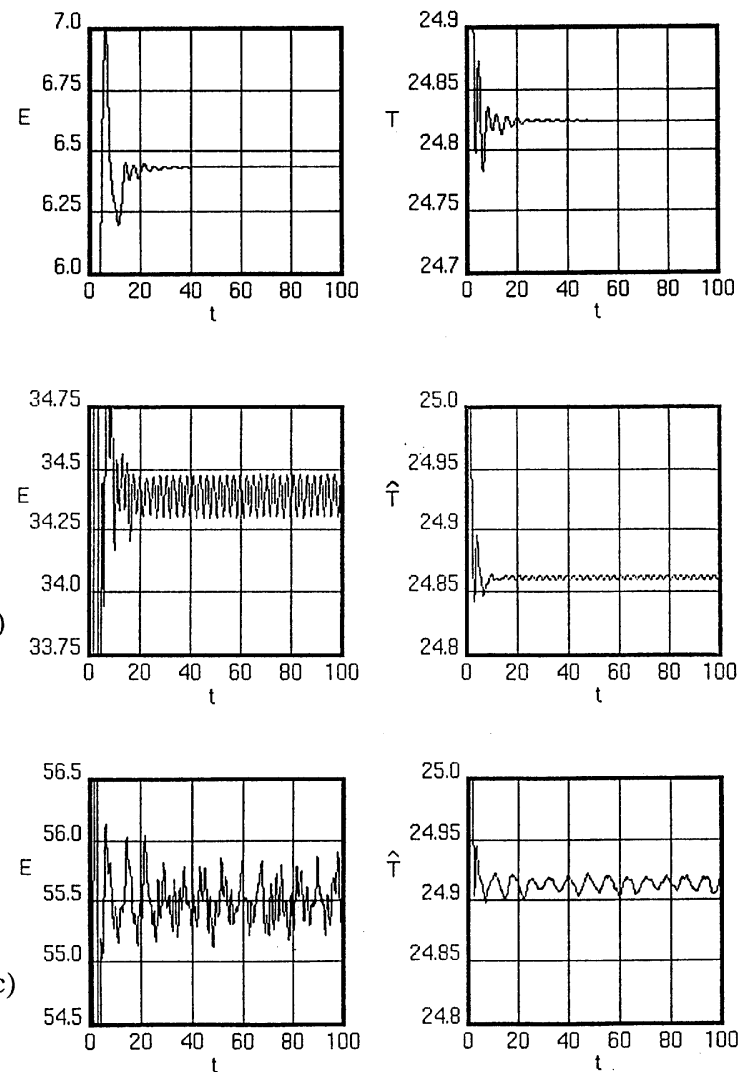

and the Taylor number Ta, with

$|\mathrm{Ta}|=4 \omega^{2} H^{4} / v^{2}$.

The Taylor number reflects the relative strength of the Coriolis acceleration. Its sign is determined by the rotation sense of centrifuge. Negative Ta corresponds to the case where $\boldsymbol{e}_{\omega}$ is parallel to the axis $z$ (i.e. the centrifuge rotation is in the same sense as the primary convection roll in the melt). Positive Ta corresponds to the case of $\boldsymbol{e}_{\omega}$ being counter-parallel to the axis $z$ (i.e. centrifuge rotation and melt convection roll are in the opposite direction).

The no slip condition is applied at the crucible walls. The "horizontal" free surface is assumed flat and stress-free, i.e.

$\boldsymbol{u}=0 \quad$ at crucible wall,

$\frac{\partial \boldsymbol{u}}{\partial \boldsymbol{n}}=0 \quad$ at free surface of $y=1.0$.
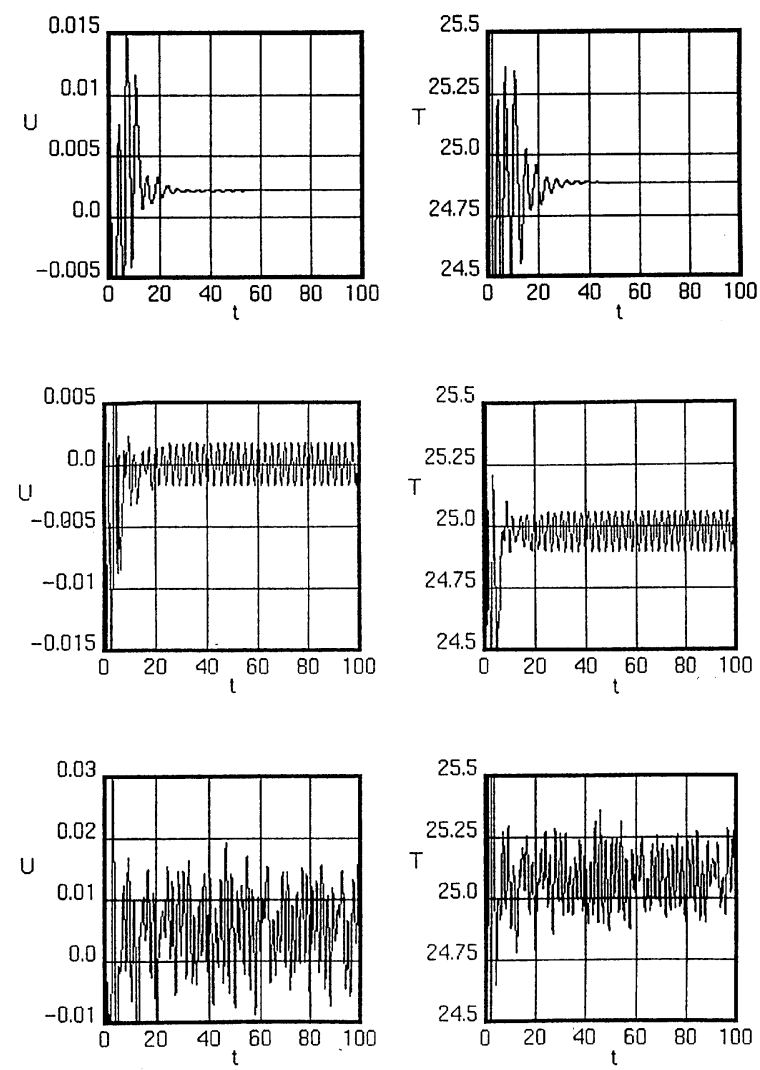

Fig. 3. The normalized total kinetic energy $E\left(10^{6}\right)$, the average temperature $\hat{T}\left({ }^{\circ} \mathrm{C}\right)$, the velocity $U(\mathrm{~m} / \mathrm{s})$ and temperature $T\left({ }^{\circ} \mathrm{C}\right)$ at the central point of the melt versus time $t$ (s) for Taylor number $\mathrm{Ta}=0$ and various Grashof numbers: $(\mathrm{a}) \mathrm{Gr}=3.8 \times 10^{6}$, (b) $\mathrm{Gr}=4.0 \times 10^{6}$, (c) $\mathrm{Gr}=7.0 \times 10^{6}$. 
(a)
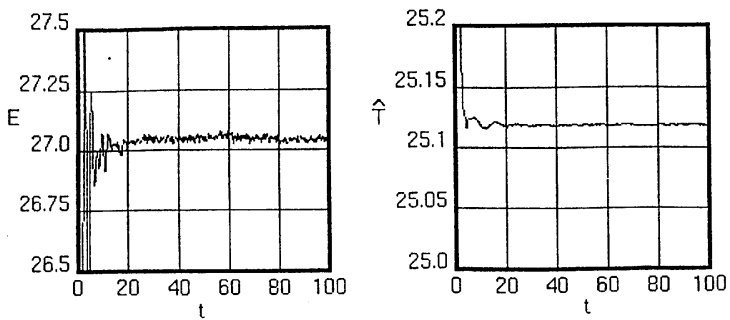

(b)

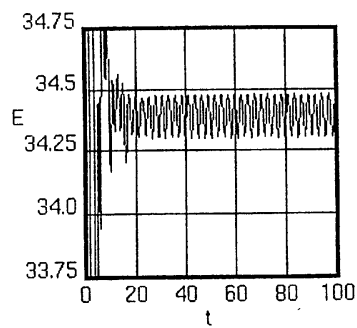

(c)

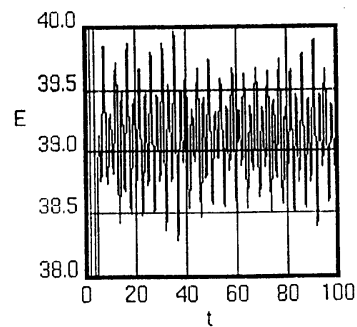

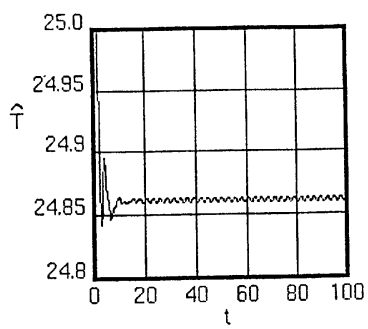
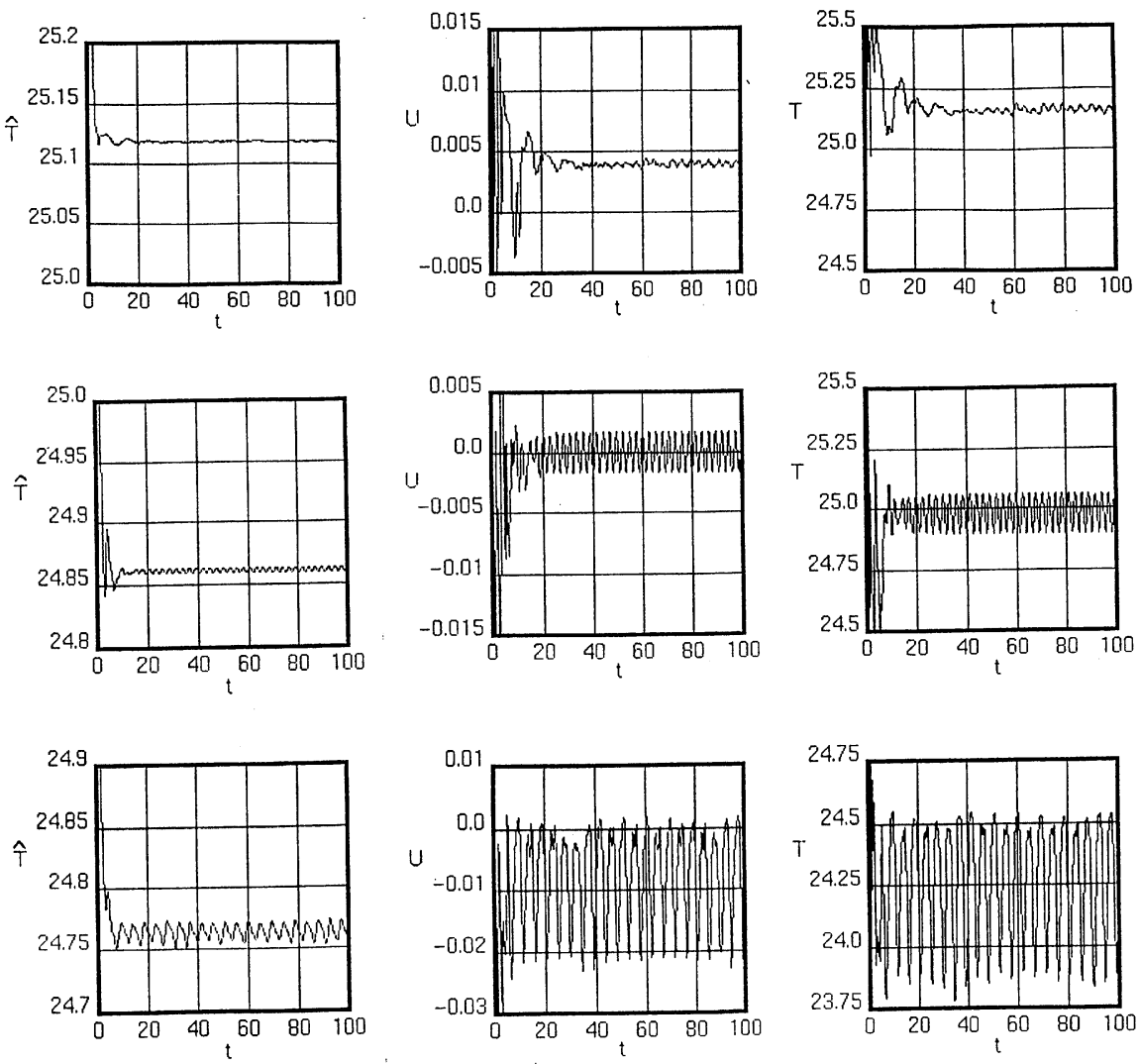

Fig. 4. The normalized total kinetic energy $E\left(10^{6}\right)$, the average temperature $\hat{T}\left({ }^{\circ} \mathrm{C}\right)$, the velocity $U(\mathrm{~m} / \mathrm{s})$ and temperature $T\left({ }^{\circ} \mathrm{C}\right)$ at the central point of the melt versus time $t(\mathrm{~s})$ for Grashof number $\mathrm{Gr}=4.0 \times 10^{6}$ and different Taylor numbers: (a) $\mathrm{Ta}=-3.6 \times 10^{5}$, (b) $\mathrm{Ta}=0$, (c) $\mathrm{Ta}=3.6 \times 10^{5}$.

The temperatures at the two end-walls are fixed at $T_{\mathrm{C}}$ and $T_{\mathrm{H}}$, respectively. Both the sidewall and the free surface are imposed with a fixed linear temperature profile.

\subsection{Numerical method}

The differential equations governing the conservation of mass, momentum, energy are discretized by the finite volume method. They are integrated over the individual computational cell, and then approximated in terms of the cell-centered nodal values of the dependent variables. This method has the advantage of ensuring that the discretized forms preserve the conservation of the original differential equations.
Implicit methods are employed to solve the algebraic finite-volume equations resulting from the discretization. The self-filter central differential scheme is applied for variables of velocity and temperature. For the time-dependent problems, the PISO algorithm (pressure implicit solution by split operator method) [12] is used to ensure reasonable transient results.

In the preliminary runs, different grid sizes are used to establish grid independence of the final results (not shown). It is found that a mesh measuring $41 \times 13 \times 11$ representing half a crucible with local refinement near the walls and free surface is deemed sufficient for the present computations. Further refinement of grids with the meshing increasing $10 \%$ in each dimension only produce 


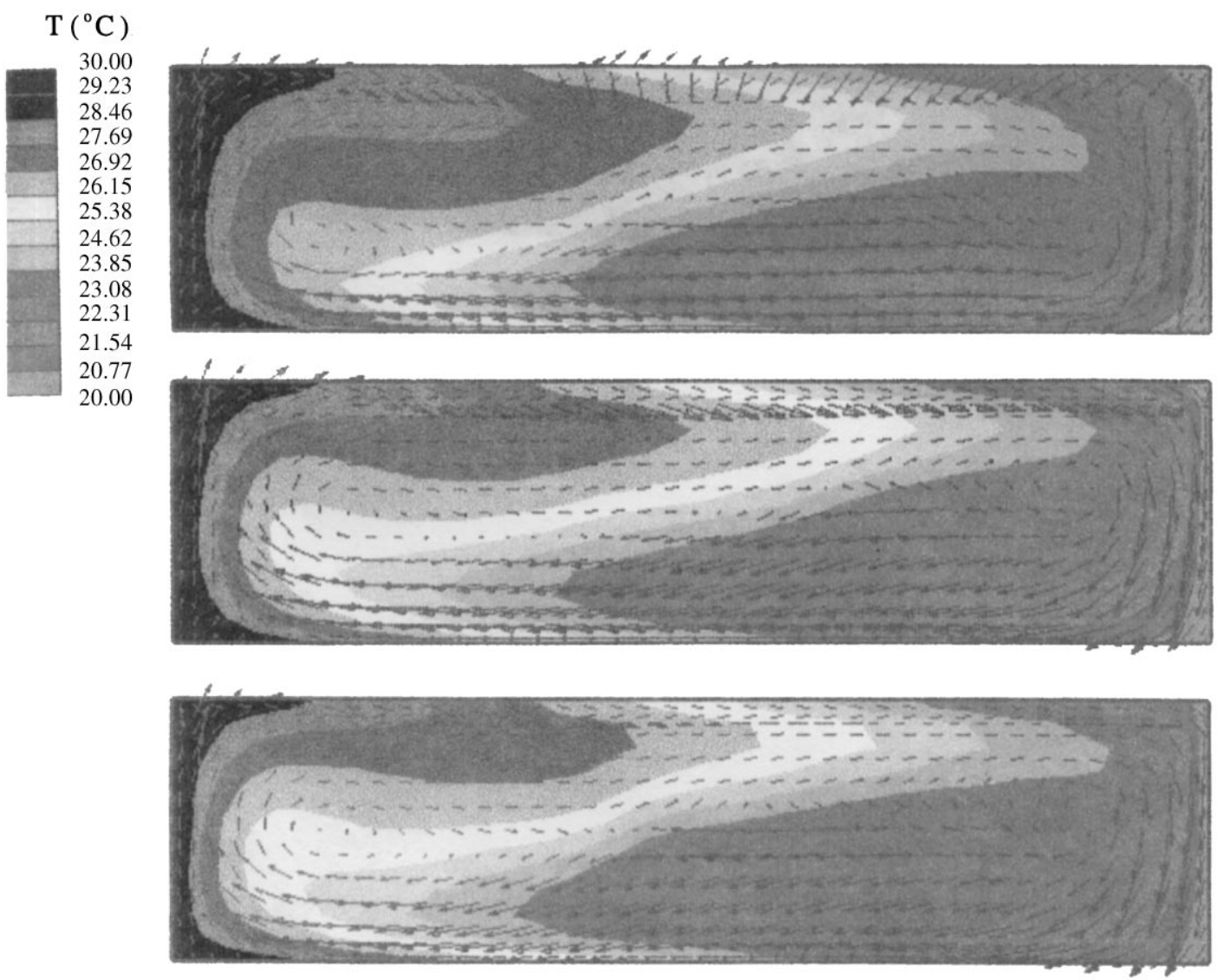

Fig. 5. The velocity vectors and the contours of temperature on the plane of symmetry at time $=100 \mathrm{~s}$ for three cases of Fig. 4. From top to bottom: $\mathrm{Ta}=-3.6 \times 10^{5}, \mathrm{Ta}=0, \mathrm{Ta}=3.6 \times 10^{5}$.

marginal difference in the results but with huge increase in computing effort. (See below the discussion on the issue of much smaller time stepping with decrease in local mesh dimension in order to maintain the prescribed Courant number.)

Since the entire geometry is symmetrical with respect to the central vertical plane, most of the calculations were undertaken utilizing half the flow domain. Runs were made occasionally with the full flow domain with almost identical results as that obtained from half the flow domain. This certainly established the validity of the assumption of flow symmetry and effectively ruled out the presence of any asymmetric three-dimensional flow features within the crucible. The time step size is taken in such a way that the Courant number,

$$
C=\frac{|U| \cdot \Delta t}{L}
$$

does not exceed certain value to meet the requirements of (a) stability of the numerical solution algorithm by minimizing the cumulative error in the numerical solution and, (b) resolution of the transient details of the flow. Here $U$ and $L$ are the characteristic velocity and dimension, respectively. (In our case: $U$ is the local velocity and $L$ is the corresponding local mesh dimension).

The calculation started from the initial condition of a stationary flow field with the temperature 

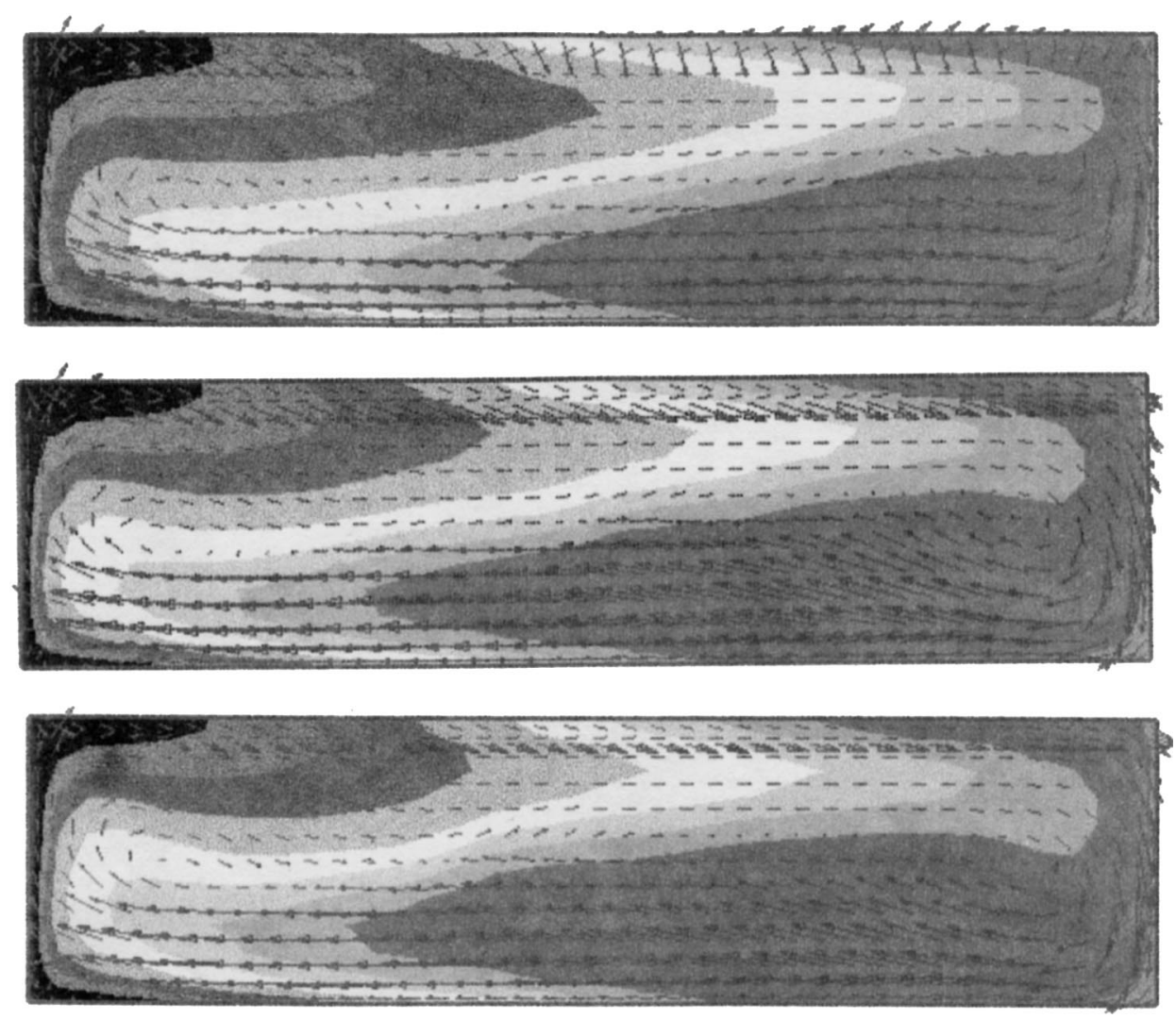

Fig. 6. The velocity vectors and the contours of temperature on the "vertical" plane of $z=0.5$ at time $=100 \mathrm{~s}$ for three cases of Fig. 4 . From top to botom: $\mathrm{Ta}=-3.6 \times 10^{5}, \mathrm{Ta}=0, \mathrm{Ta}=3.6 \times 10^{5}$.

$T=0$ everywhere. The calculations were repeated for various values of $\mathrm{Gr}$ and Ta to seek the critical state of an oscillatory motion and optimal stabilizing conditions.

\section{Numerical results and discussion}

The time-dependent behavior of the melt flow in three dimensions can be described by the nondimensional total kinetic energy

$E=\frac{1}{2} \int_{\Omega}\left(u^{2}+v^{2}+w^{2}\right) \mathrm{d} \Omega$,

where $\Omega$ is the nondimensional flow domain, and $(u, v$ and $w$ ) denote the nondimensional velocity components in the $(x, y, z)$ coordinate system. $E$ reflects the strength of the melt convection. We define the average temperature over the entire domain of the melt as

$\hat{T}=\frac{1}{\Omega} \int_{\Omega} T \mathrm{~d} \Omega$.

First, we computed the cases without centrifuge rotation (i.e. with $\mathrm{Ta}=0$ ) but taken into account the effect of the gravity. Fig. 3 shows that the flow is steady at low Grashof number (i.e. $\mathrm{Gr}=3.8 \times 10^{6}$ ). With the increase of $\mathrm{Gr}$, the flow indicates a tendency to oscillate periodically. Both the magnitude and amplitude of fluctuation of the total kinetic energy $E$ also increase progressively. At even higher $\mathrm{Gr}$, it exhibits irregular oscillations. In this simulation the critical value of $\mathrm{Gr}$ where the regular oscillation persists is located between $7 \times 10^{5}$ and $8 \times 10^{5}$. This is discernibly larger than the result based on two-dimensional simulation [11], where 

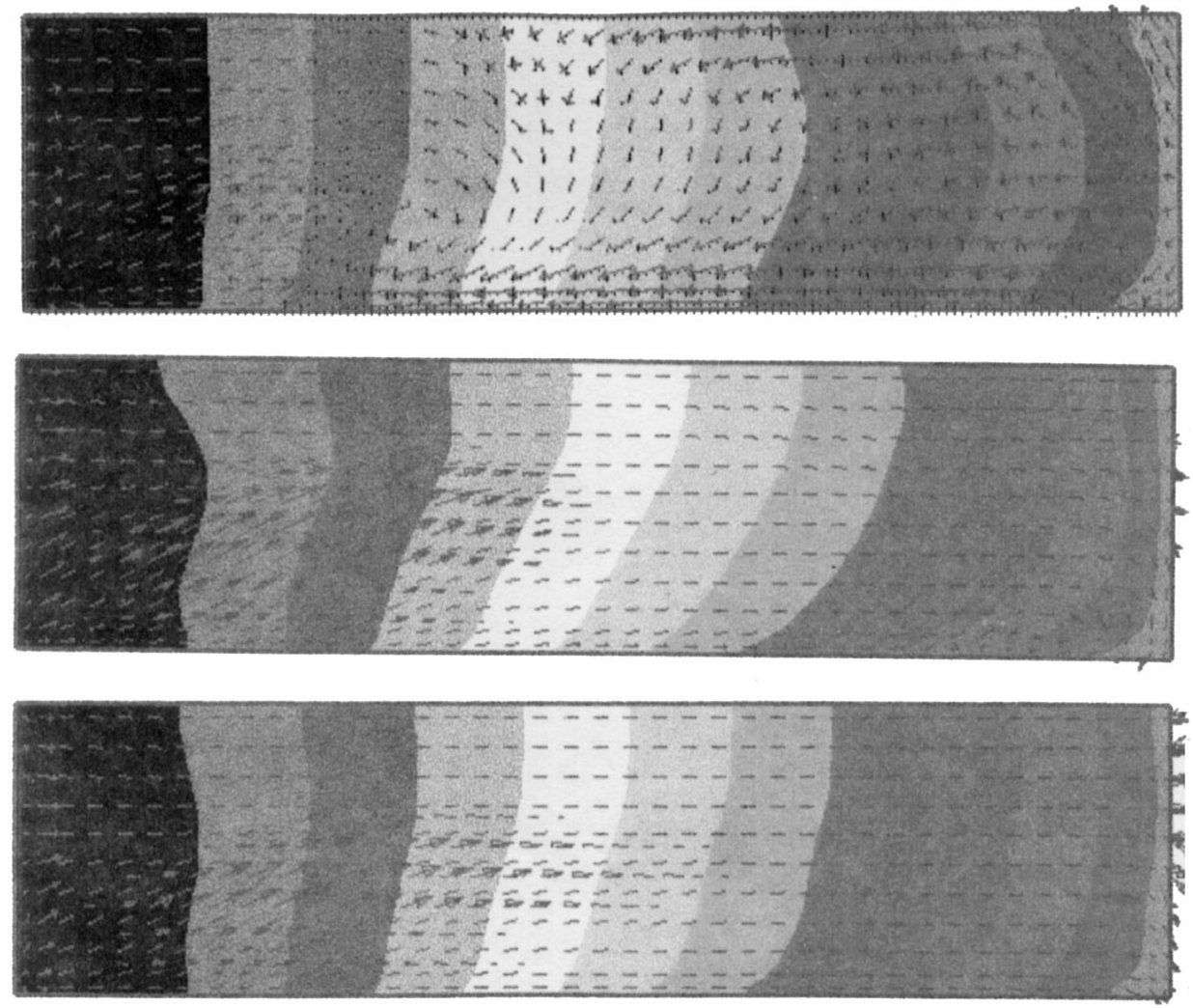

Fig. 7. The velocity vectors and the contours of temperature on the free surface at time $=100 \mathrm{~s}$ for three cases of Fig. 4 . From top to bottom: $\mathrm{Ta}=-3.6 \times 10^{5}, \mathrm{Ta}=0, \mathrm{Ta}=3.6 \times 10^{5}$.

the critical value of $\mathrm{Gr}$ is determined to be less than $5.5 \times 10^{5}$.

It is obvious from Fig. 3 that the evolution of the average temperature $\hat{T}$, the velocity $U$ and temperature $T$ at the central point of the melt are similar to the behavior of total kinetic energy $E$.

When the centrifuge is in rotation $(\mathrm{Ta} \neq 0)$, the Coriolis effect becomes apparent. The obtained results have dramatically changed from the case of $\mathrm{Ta}=0$. Fig. 4a-Fig. $4 \mathrm{c}$ show the evolutions of the total kinetic energy $E$, the average temperature $\hat{T}$, the velocity $U$ and temperature $T$ at the central point of the melt for a given Grashof number $\left(\mathrm{Gr}=4 \times 10^{6}\right)$ and different Taylor numbers of $\mathrm{Ta}=0, \mathrm{Ta}=-3.6 \times 10^{5}$ and $\mathrm{Ta}=3.6 \times 10^{5}$, respectively. It is obvious that the oscillations of the kinetic energy are largely suppressed for $\mathrm{Ta}=-3.6 \times 10^{5}$. On the other hand, for $\mathrm{Ta}=3.6 \times 10^{5}$, there is an even larger oscillation observed compared to $\mathrm{Ta}=0$. In addition, the intensity of the oscillations for the convective velocity is lower for the case of $\mathrm{Ta}=-3.6 \times 10^{5} \mathrm{com}-$ pared to the cases $\mathrm{Ta}=0$ and $\mathrm{Ta}=3.6 \times 10^{5}$. Again, the temperature $\hat{T}$ and $T$ indicate a very similar behavior.

This suggests that the Coriolis force has a profound influence on the bulk flow in the melt. The most important and critical factor is the sign of $\mathrm{Ta}$ (e.g. the rotation sense of the centrifuge), which determines the different actions of Coriolis accelerations acting on the convective flows in the melt in the presence of Coriolis accelerations which are of identical magnitude. This result clearly indicates the stabilizing influence of negative $\mathrm{Ta}$ on the bulk flow, which is in full agreement with the experiments by Ma et al. [1] and Müller [13].

Figs. 5-8 present the velocity vectors and the contours of temperature in the various cross 

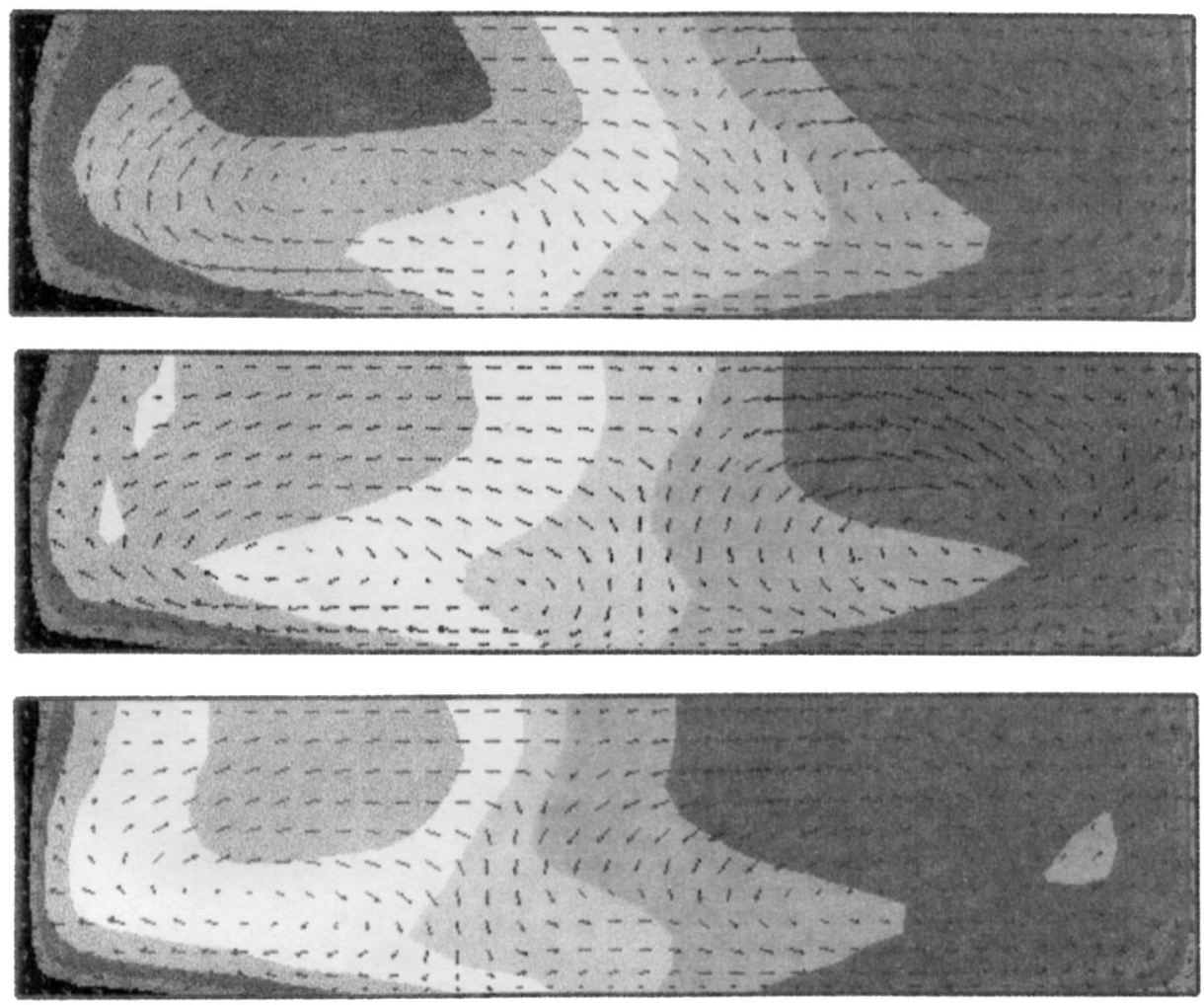

Fig. 8. The velocity vectors and the contours of temperature on the "horizontal" plane of $y=0.5$ at time $=100 \mathrm{~s}$ for three cases of Fig. 4. From top to bottom: $\mathrm{Ta}=-3.6 \times 10^{5}, \mathrm{Ta}=0, \mathrm{Ta}=3.6 \times 10^{5}$.

sections of the melt at time $=100 \mathrm{~s}$ for the three cases $\mathrm{Ta}=-3.6 \times 10^{5}, \mathrm{Ta}=0$ and $\mathrm{Ta}=3.6 \times 10^{5}$ corresponding to Fig. 4. Convective flow structures can be observed. These figures clearly show that the flow pattern for the case of negative Ta is quite different from those for the other two cases either in the plane of symmetry (Fig. 5) or on the "vertical" plane of $z=0.5$ (Fig. 6). The three-dimensional effect is further seen by comparing the flow features on the free surface ( $y=1.0$, i.e. Fig. 7) and on the "horizontal" plane of $y=0.5$ (Fig. 8). Eddies can be seen on the horizontal plane view. On the other hand, in two-dimensional simulation $[11,14]$, the multicellular pattern of flow structure is only detected in the vertical section plane and at Gr number greater than the critical value of $5.5 \times 10^{5}$.

The spectra of total kinetic energy $E$ obtained by Fourier transformation are given in Fig. 9. It can be seen that the maximum peak of the energy spectra which is evident at the low frequency of about $0.38 \mathrm{~Hz}$ for $\mathrm{Ta}=0$ is suppressed for the case $\mathrm{Ta}=-3.6 \times 10^{5}$. For $\mathrm{Ta}=3.6 \times 10^{5}$, the maximum peak has shifted marginally to a higher frequency at about $0.41 \mathrm{~Hz}$. This is already obvious from Fig. 4. The second spectra peak observed at $f=0.77 \mathrm{~Hz}$ for $\mathrm{Ta}=0$ behaves in a fairly similar fashion as for the first peak when $\mathrm{Ta}$ is changed accordingly.

Taking the square root of the variance of $E$ as a measure of the strength of the flow oscillations (denoted by $\bar{E}^{\prime}$ ), we plot $\bar{E}^{\prime}$, as well as $\bar{E}$ (the mean value of total kinetic energy) and $\overline{\hat{T}}$ (the mean value of average temperature) against $\sqrt{|\mathrm{Ta}|}$ in Fig. 10. (The over-bar denotes the mean values calculated over the period of the evolution time. $\sqrt{|\mathrm{Ta}|}$ is proportional to the rotation rate of the centrifuge 

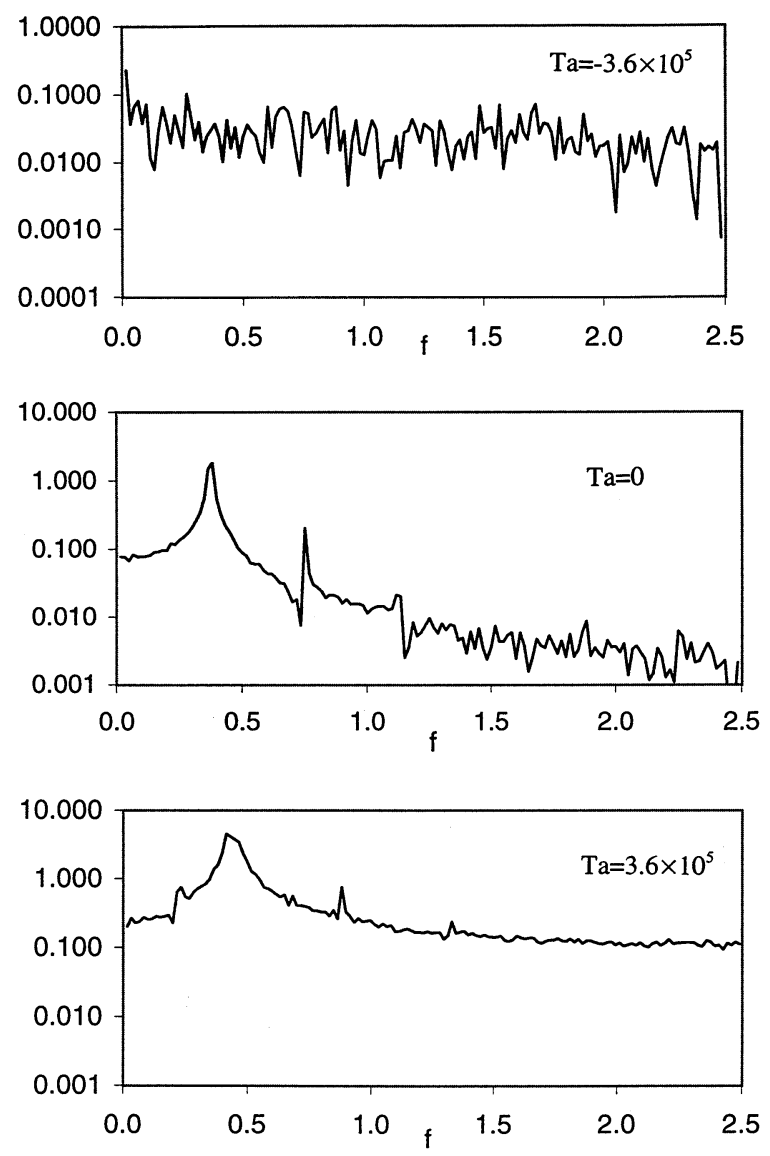

Fig. 9. The spectra of total kinetic energy $E\left(\mathrm{Gr}=4.0 \times 10^{6}\right)$.

according to the definition of Taylor number.) From Fig. 10, the following features are noted:

(1) For different rotation directions of the centrifuge (i.e. for different signs of Ta) the profiles of $\bar{E}^{\prime}$ are obviously different. $\bar{E}^{\prime}$ is significantly larger for the cases of positive Ta in comparison to negative Ta. This indicates that, generally, the condition of negative $\mathrm{Ta}$ is favorable for the reduction or damping of possible flow oscillations. Nevertheless, there are certain rotation rates near $\sqrt{|\mathrm{Ta}|}=200$ and $\sqrt{|\mathrm{Ta}|}=700$ in Fig. 10a, where $\bar{E}^{\prime}$ indicates a smaller quantity than for the nonrotation case. Furthermore, for the cases of negative Ta, there are also some data points in Fig. 10b for which $\bar{E}^{\prime}$ exhibits a magnitude larger than that for nonrotation case; when $\sqrt{|\mathrm{Ta}|}$ exceeds about $1000, \bar{E}^{\prime}$ becomes considerably larger.
All these results correspond fairly well with the results of Ma et al. [1] and Müller et al. [13]. According to them, for the condition of positive Ta the Coriolis force should act inwards with respect to the convection roll, hence pushing the fluid towards the center of the convection roll and causing the flow to become more unstable. For the condition of negative Ta, the Coriolis force acts outwards with respect to the convection roll, thereby forcing the fluid to stream along elongated paths. As a result, convective heat transfer is increased and the flow oscillations are damped. Fig. 11 illustrates the two patterns of Coriolis effects based on the postulation (from Ref. [1]).

It may be mentioned that in the experiments of Ma et al. [1], no marked variation of the amplitudes of the temperature fluctuations was found for the negative Ta conditions as the centrifugal acceleration varies. This discrepancy with our simulations (see Fig. 10b) can be probably attributed to the different thermal boundary conditions between the numerical model and the experiments. In the former, the thermal boundary condition is assumed as a linear temperature profile with a fixed longitudinal temperature gradient. In the experiments, the longitudinal temperature gradient cannot be fixed; it may decrease as the thermal convection is enhanced due to the increasing rotation rate. Accordingly, the thermal boundary conditions on the crucible surface between the simulations and experiments are different.

It would be interesting to verify experimentally the aspect of the computed results which predict that under certain parametric cases of positive Ta there is a suppression of flow oscillations, and for very large negative Ta the flow becomes more unstable.

(2) The mean value of total kinetic energy $(\bar{E})$, increases linearly with increasing $\sqrt{|\mathrm{Ta}|}$, while the mean value of average temperature $(\overline{\hat{T}})$, decreases linearly for the case of positive Ta (see Fig. 10a). This suggests that the presence of the Coriolis force has the overall resultant effect of "converting" the internal energy into kinetic energy in the melt flow. For the cases of negative Ta, one finds the reverse trends of $\bar{E}$ and $\bar{T}$ versus $\sqrt{|\mathrm{Ta}|}$. The latter indicates that the Coriolis effect manifests as the "conversion" of kinetic energy into internal 

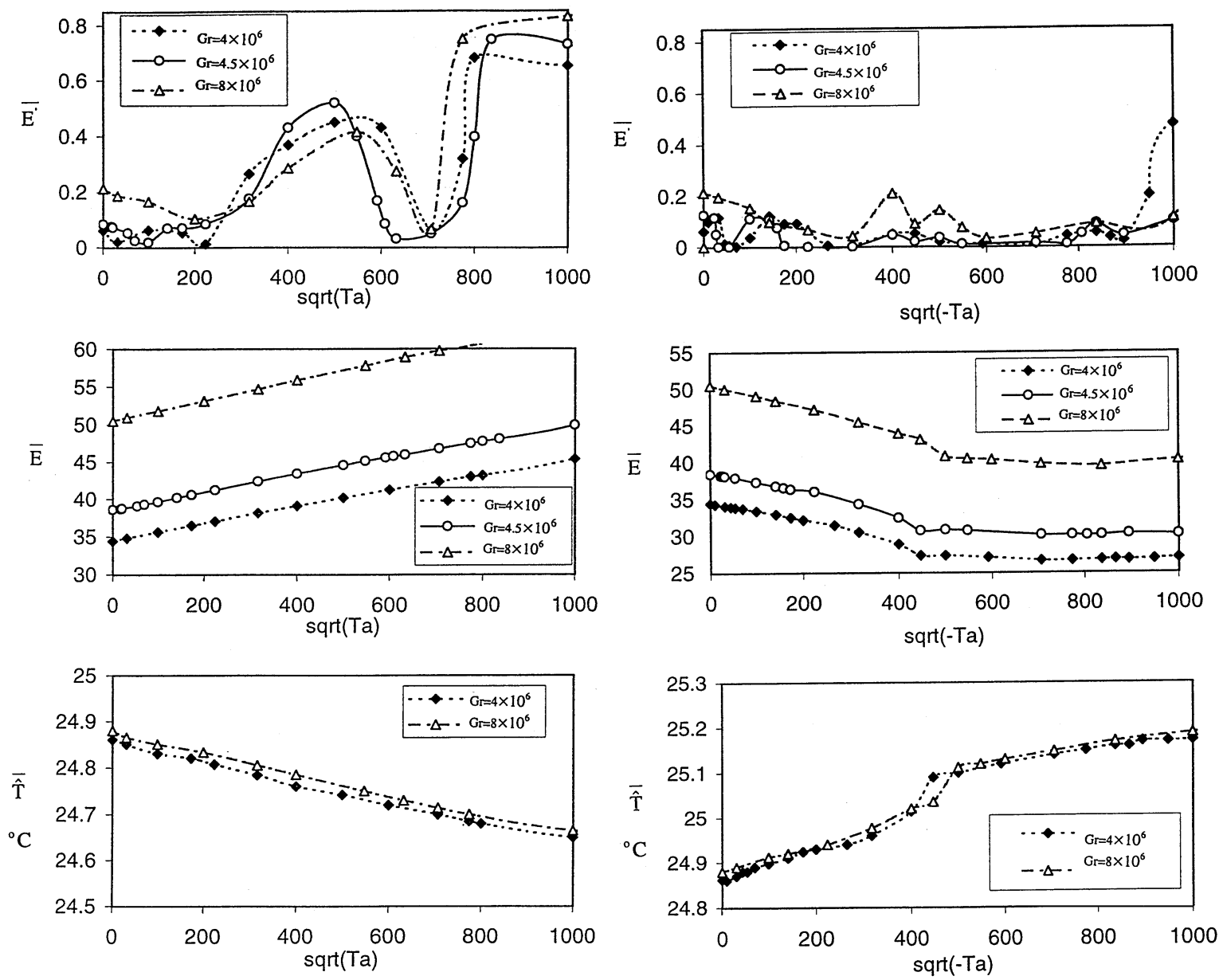

(a)

(b)

Fig. 10. (a) Plot of the strength of flow oscillation $\bar{E}^{\prime}$, the mean value of total kinetic energy $\bar{E}$ and the mean value of the average temperature $\overline{\hat{T}}$ against $\sqrt{|\mathrm{Ta}|},(\mathrm{Ta}>0)$. (b) Plot of the strength of flow oscillation $\bar{E}^{\prime}$, the mean value of total kinetic energy $\bar{E}$ and the mean value of the average temperature $\bar{T}$ against $\sqrt{|\mathrm{Ta}|},(\mathrm{Ta}>0)$.

energy in the melt. Such co-relation exists between $E$ and $\hat{T}$ as well. One can find this behavior in Fig. 4.

Finally, it may be observed that there is a slight "kink" in the distribution of $\bar{E}$ and $\bar{T}$ versus $\sqrt{|\mathrm{Ta}|}$ for negative $\mathrm{Ta}$ in the vicinity of $\sqrt{|\mathrm{Ta}|}=450$. Preliminary examinations of the computed flow and temperature fields do not yield any apparent reason for the occurrence. Perhaps some experimental works could be done to confirm the trend and behavior.

\section{Conclusion}

Three-dimensional and time-dependent numerical simulations for the bulk flows during horizontal Bridgman crystal growth on a centrifuge were carried out using the finite volume method. As observed in previous experiments the results of the numerical simulations show the importance of Coriolis force, which is generated due to the rotation of centrifuge. The Coriolis force stabilizes the oscillatory motion in the melt under some particular 

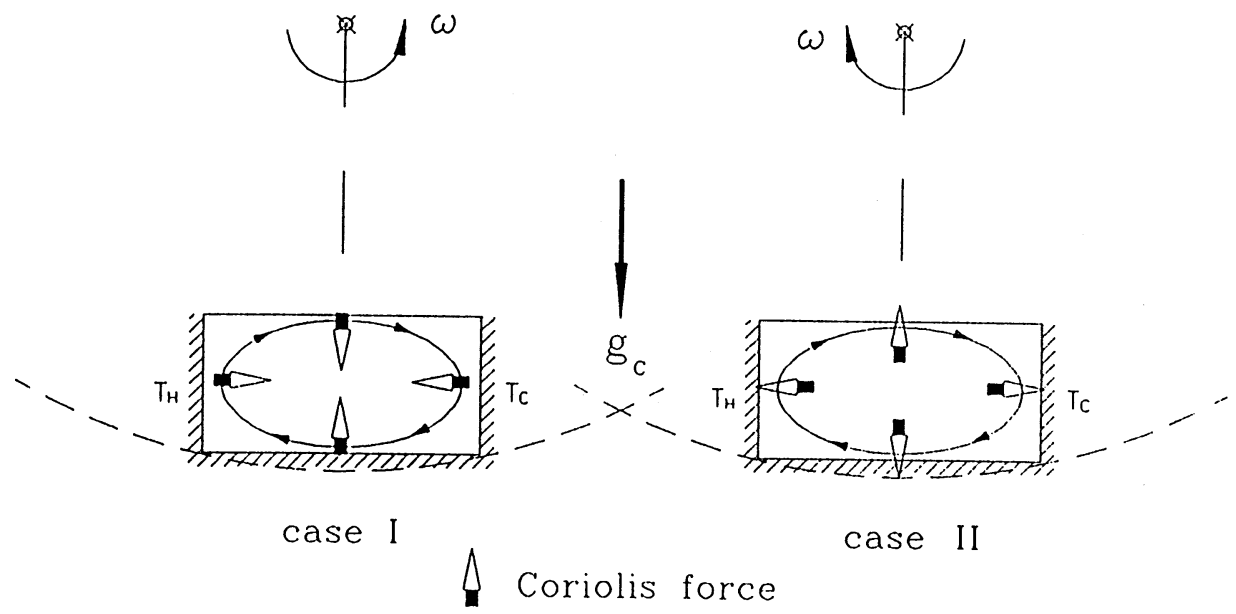

Fig. 11. Illustration of the two patterns of Coriolis effects (from Ref. [13]).

operating conditions of the centrifuge, which can be summarized as follows:

(a) Counter-rotation flow (i.e. $\mathrm{Ta}>0$ ) is not conducive for damping of flow oscillations. When rotation rate exceeds a certain value, the flow becomes more unstable.

(b) Iso-rotation flow (i.e. Ta $<0$ ) is favorable for the reduction or damping of possible flow oscillations. Although the general condition of similar rotation direction of both the centrifuge and the melt convection roll is more favorable for damping of the flow oscillation, the precise nature of influence of Coriolis force depends on the actual combination of rotating rate and rotation direction of the centrifuge.

Further analysis of the computed results show that with Ta $>0$, the Coriolis effect acts to increase the kinetic energy of the melt flow while its internal energy decreases. On the other hand, with increasing magnitude of $\mathrm{Ta}(<0)$, the Coriolis effect manifests as the "conversion" of kinetic energy into internal energy thereby resulting in the raising of the overall melt temperature.

\section{Acknowledgements}

W.J. Ma wishes to express his sincere appreciation to the National University of Singapore for hosting his visit at the Institute of High Performance Computing. The authors would like to thank Professor M.L. Xue and Dr. C. Shu for helpful discussions.

\section{References}

[1] W.J. Ma, F. Tao, Y. Zheng, M.L. Xue, B.J. Zhou, L.Y. Lin, Response of temperature oscillations in a tin melt to Centrifugal effects, in: L.L. Regel, W.R. Wilcox (Eds.), Materials Processing in High Gravity, Plenum Press, New York, 1994, p. 61.

[2] G. Müller, Convection and inhomogeneities, in: Crystal Growth from the Melt Crystals, vol. 12, Springer, Berlin, 1988.

[3] H. Rodot, L.L. Regel, G.V. Sarafanov, H. Hamidi, I.V. Videskii, A.M. Turtchaninov, J. Crystal Growth 79 (1986) 77.

[4] H. Rodot, L.L. Regel, A.M. Turtchaninov, J. Crystal Growth 104 (1990) 280.

[5] G. Müller, P. Kyr, E. Schmidt, J. Crystal Growth 49 (1980) 387.

[6] G. Müller, G. Neumann, J. Crystal Growth 59 (1982) 548.

[7] X.R. Zhong, B.J. Zhou, Q.M. Yan, F.N. Cao, C.J. Li, L.Y. Lin, W.J. Ma, Y. Zheng, F. Tao, M.L. Xue, J. Crystal Growth 119 (1992) 74.

[8] B.J. Zhou, F.N. Cao, L.Y. Lin, W.J. Ma, Y. Zheng, F. Tao, M.L. Xue, Growth of GaAs single crystals under high gravity condition, in: L.L. Regel, W.R. Wilcox (Eds.), Materials Processing in High Gravity, Plenum Press, New York, 1994, p. 53. 
[9] G. Neumann, J. Fluid Mech. 214 (1990) 559.

[10] N. Ramechandran, J.P. Downey, P.A. Curreri, J.C. Jones, J. Crystal Growth 126 (1993) 655.

[11] F. Tao, Y. Zheng, W.J. Ma, M.L. Xue, Unsteady thermal convection of melts in a 2-D horizontal boat in a centrifugal field with consideration of the Coriolis effect, in: L.L. Regel, W.R. Wilcox (Eds.), Materials Processing in High Gravity, Plenum Press, New York, 1994, p. 67.
[12] R.I. Issa, A.D. Gosman, A.P. Watkins, J. Comp. Phys. 62 (1986) 66.

[13] G. Müller, G. Neumann, W. Weber, J. Crystal Growth 119 (1992) 8.

[14] S. Dupont, J.M. Marchal, M.J. Crochet, Int. J. Numer. Meth. Fluids 7 (1987) 49. 